\title{
Properties of a bifurcated current sheet observed on 29 August 2001
}

\author{
A. Runov $^{1}$, V. Sergeev ${ }^{2}$, R. Nakamura ${ }^{1}$, W. Baumjohann ${ }^{1}$, Z. Vörös ${ }^{1}$, M. Volwerk ${ }^{1,3}$, Y. Asano ${ }^{1}$, B. Klecker ${ }^{3}$, \\ H. Rème ${ }^{5}$, and A. Balogh 6 \\ ${ }^{1}$ Institut für Weltraumforschung der ÖAW, Graz, Austria \\ ${ }^{2}$ St. Petersburg University, Russia \\ ${ }^{3}$ MPE Garching, Germany \\ ${ }^{5}$ CESR, Toulouse, France \\ ${ }^{6}$ Imperial College, London, UK
}

Received: 4 September 2003 - Revised: 15 March 2004 - Accepted: 20 April 2004 - Published: 14 July 2004

Part of Special Issue "Spatio-temporal analysis and multipoint measurements in space"

\begin{abstract}
Ion density, velocity and temperature, measured by Cluster spacecraft in the plasma sheet during an isolated substorm at 10:20-11:15 UT, 29 August 2001 are discussed. The Custer/CODIF data for subinterval 10:55-11:03 UT, during which a bifurcation of the current sheet was detected, are studied in particular. It is shown that ion density and temperature have a plateau-like profile in the central part of the bifurcated current sheet. An enhanced proton flow directed in positive $Y_{\mathrm{GSM}}$ was detected during the selected subinterval. Other components of proton bulk velocity are found to be negligibly small, i.e. the structure is likely not directly associated with magnetic reconnection. Alternative models, including ion anisotropy and localized magnetic turbulence, which result in a two-peak profile of the current density, are discussed briefly.
\end{abstract}

Key words. Magnetospheric physics (magnetotail, plasma sheet)

\section{Introduction}

Observations of the magnetotail current sheet and theoretical studies of current sheet equilibrium and evolution show that the spatial structure of the current sheet is often different from the simple 1-D model (Harris, 1962) with a broad maximum of current density in the sheet center, and can include a strong current in the central region (embedded thin current sheets, see, e.g. Sitnov et al., 2000) or more complicated distributions with double off-center peaks, i.e. bifurcated current sheets (see, e.g. Delcourt and Belmond, 1998, and references therein).

The observational evidence for bifurcated current sheets is still very scarce because of difficulties to reconstructing the spatial profile of the magnetic shear in a time varying config-

Correspondence to: A. Runov

(andrei.runov@oeaw.ac.at) uration with a single spacecraft. Previous studies with ISEE$1 / 2$ dual s/c system gave only one example of a bifurcated thin current sheet (Sergeev et al., 1993). Other arguments for the existence of bifurcated sheets (indirect, based primarily on the $B$-field occurrence distribution) have been given by Hoshino et al. (1996), although they refer to the distant tail plasma sheet, where the properties are different from those in the near- and mid-tail regions. Further evidence of current bifurcation have been recently obtained by Asano et al. (2003), who analyzed the current densities obtained from ion and electron velocities measured by the Geotail spacecraft.

Using four-point magnetic field measurements by Cluster spacecraft, Nakamura et al. (2002) found a current sheet with an off-center (displaced from $B_{x}=0$ ) peak of the magnetic field gradient during fast earthward flow. Runov et al. (2003) studied the orientation and structure of the current sheet during 10:55-11:07 UT on 29 August 2001 by the analysis of multi-point magnetic field measurements. Using the Cluster tetrahedron configuration the vertical magnetic field gradient was estimated as a difference between $X_{\mathrm{GSM}}$ components of the magnetic field, measured by three spacecraft, forming a triangle in a plane approximately parallel to the magnetic equator and fourth spacecraft, located $1700 \mathrm{~km}$ southward. It was shown that during 10:55-11:03 UT the vertical magnetic field gradient has a maximum value at mean $B_{x}$ of a half lobe value and minimum at $\left\langle B_{x}\right\rangle \sim 0$. Assuming the northsouth symmetry of the current sheet we concluded that during this interval the current sheet is bifurcated (two current layers separated by a layer of weak uniform magnetic field).

Recently, Sergeev et al. (2003) reported on another example of a bifurcated current sheet, observed by Cluster on 26 September 2001. Again, the magnetic field gradient (or current density) has two maxima at $\left\langle B_{x}\right\rangle \sim \pm 0.5 B_{L}$ and a broad minimum in between. It was shown that the plasma density has a plateau profile between the peaks of the enhanced magnetic field gradient. The analysis of the magnetic field measurements' occurrence over the current sheet crossing 


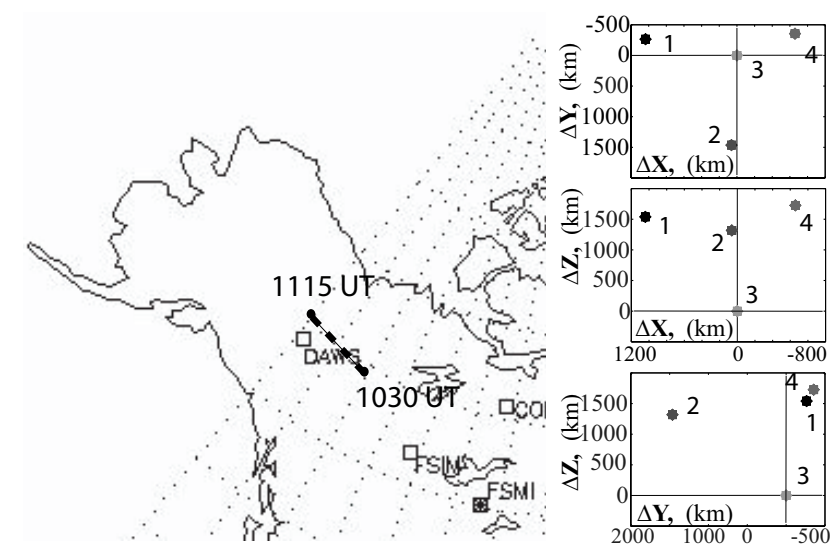

Fig. 1. Geometric projection of the Cluster orbit, calculated using T96 model, and the Cluster tetrahedron configuration at 10:2011:15 UT, 29 August 2001 (day 241).

showed that the spacecraft stay longer near $\left\langle B_{x}\right\rangle \sim 0$, which proves that the magnetic field gradient in this region is weak.

In the cases of 29 August and 26 September 2001, the current sheet bifurcations were associated with intensive northsouth motion of the current sheet, caused by wave transients passing by Cluster in the $Y_{\mathrm{GSM}}$ direction. The minimum variance and multi-point timing analysis showed that the current sheet normal rotates in the $Y-Z$ plane with an amplitude of $40-60^{\circ}$. Since on 26 September Cluster crossed the current sheet from $B_{x} \sim 25$ to $B_{x} \sim-25 \mathrm{nT}$, the separate analysis of the orientation in the northern half and southern one showed that this wave is a kink-like one. The quasi-period of the wave in both the 29 August and 26 September events was found to be of 90-100 s.

In this paper we extend the analysis by Runov et al. (2003) of the bifurcated current sheet, observed by Cluster on 29 August 2001, using solar wind data, ground-based data and data from the Cluster/CIS (Rème et al., 2001) experiment to i) establish a correspondence between the bifurcated current sheet observation and the substorm phase, ii) to analyze the plasma properties within the bifurcated current sheet, and iii) to compare these features for the 29 August 2001 and 26 September 2001 cases. Some relevant theoretical models are reviewed in the Discussion.

\section{August, 10:20-11:15 UT: Data overview}

During 10:20-11:15 UT Cluster was located at $(-19.3,-1.2,1.2) R_{E}$ (GSM coordinate system is used throughout this paper). The corresponding geographic projection of the Cluster trajectory (T96 model is used) and the Cluster tetrahedron configuration with the s/c\#3 as a reference point are shown in Fig. 1. Figure 2 shows the $y$ - and $z$-components of the interplanetary magnetic field and $x$-component of the solar wind velocity from ACE spacecraft, located around the L1 libration point, the $H$-components of the CANOPUS network magnetometer

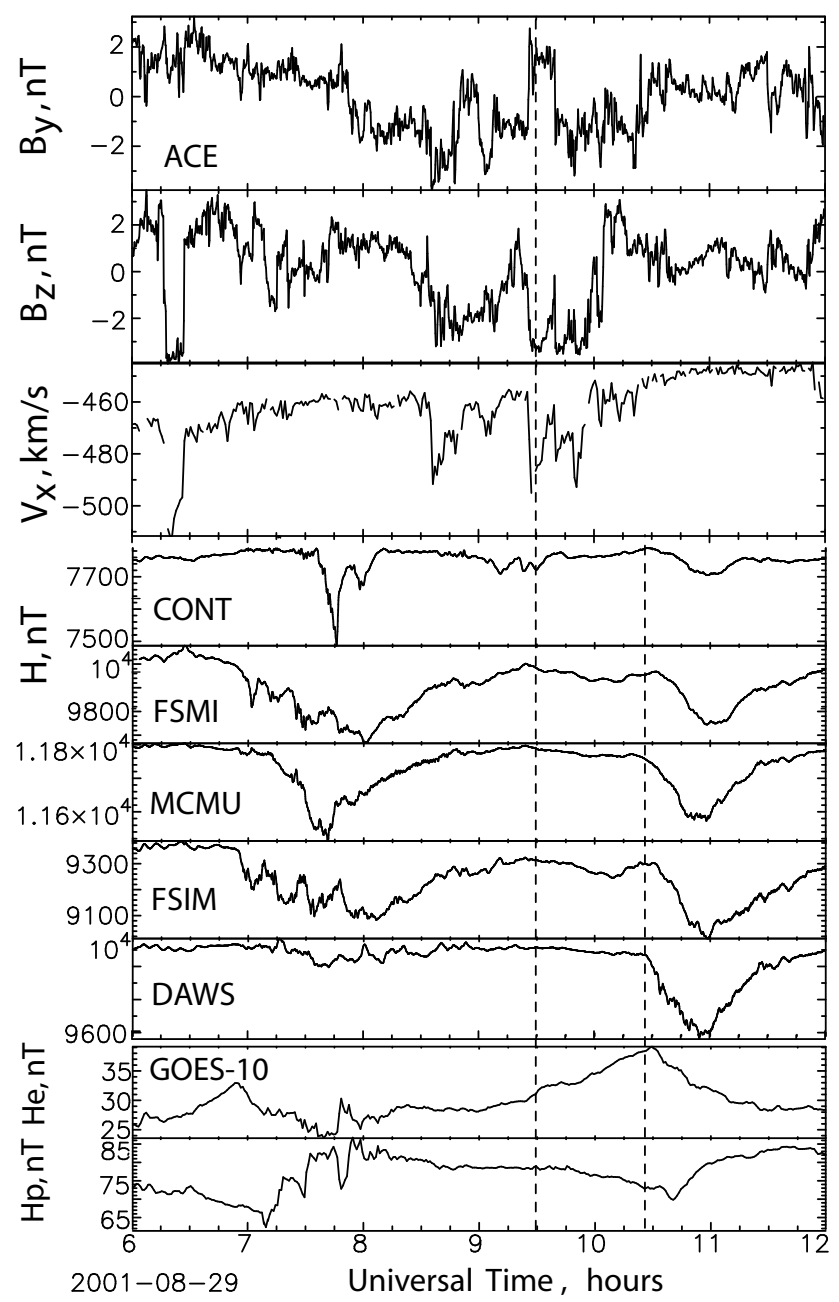

Fig. 2. Solar wind parameters from ACE: $X, Y, Z$ (GSE) IMF components, proton number density, $X$-component of proton bulk velocity, and ground-based magnetometer data from CANOPUS network (Dawson) for 06:00-12:00 UT, 29 August 2001. Dashed lines display the time shift of $53 \mathrm{~min}$ between ACE and ground-based data.

records, $H_{e^{-}}$and $H_{p}$-components from the GOES-10 spacecraft during 06:00-12:00 UT.

The CANOPUS record shows the westward auroral electrojet activation at 10:28 UT. The maximum negative bay amplitude is of $500 \mathrm{nT}$ (Dawson). According to ACE data the solar wind was about $-460-480 \mathrm{~km} / \mathrm{s}$, which gives a transport time from the L1 point of 52-55 min. The isolated substorm with onset at 10:28 UT was caused by a solar wind acceleration accompanied by negative IMF $B_{z}(-3 \mathrm{nT})$ and positive $B_{y}(\sim 2 \mathrm{nT})$ variations at 09:30-09:37 UT.

The GOES-10 spacecraft, located at the nightside detects an increase in the $H_{e}$-component from $28 \mathrm{nT}$ to $39 \mathrm{nT}$, starting at 09:00 UT, which corresponds to magnetotail stretching during the substorm growth phase, and a decrease in $H_{e}$ at $\sim 10: 30 \mathrm{UT}$, followed by an increase in $H_{p}$ from $70 \mathrm{nT}$ to $85 \mathrm{nT}$ at $\sim 10: 40 \mathrm{UT}$, which is a signature of dipolarization. At $\sim 10: 35$ UT and at $\sim 10: 45$ UT short-time injections of particles were detected by the LANL 1991-080 spacecraft 


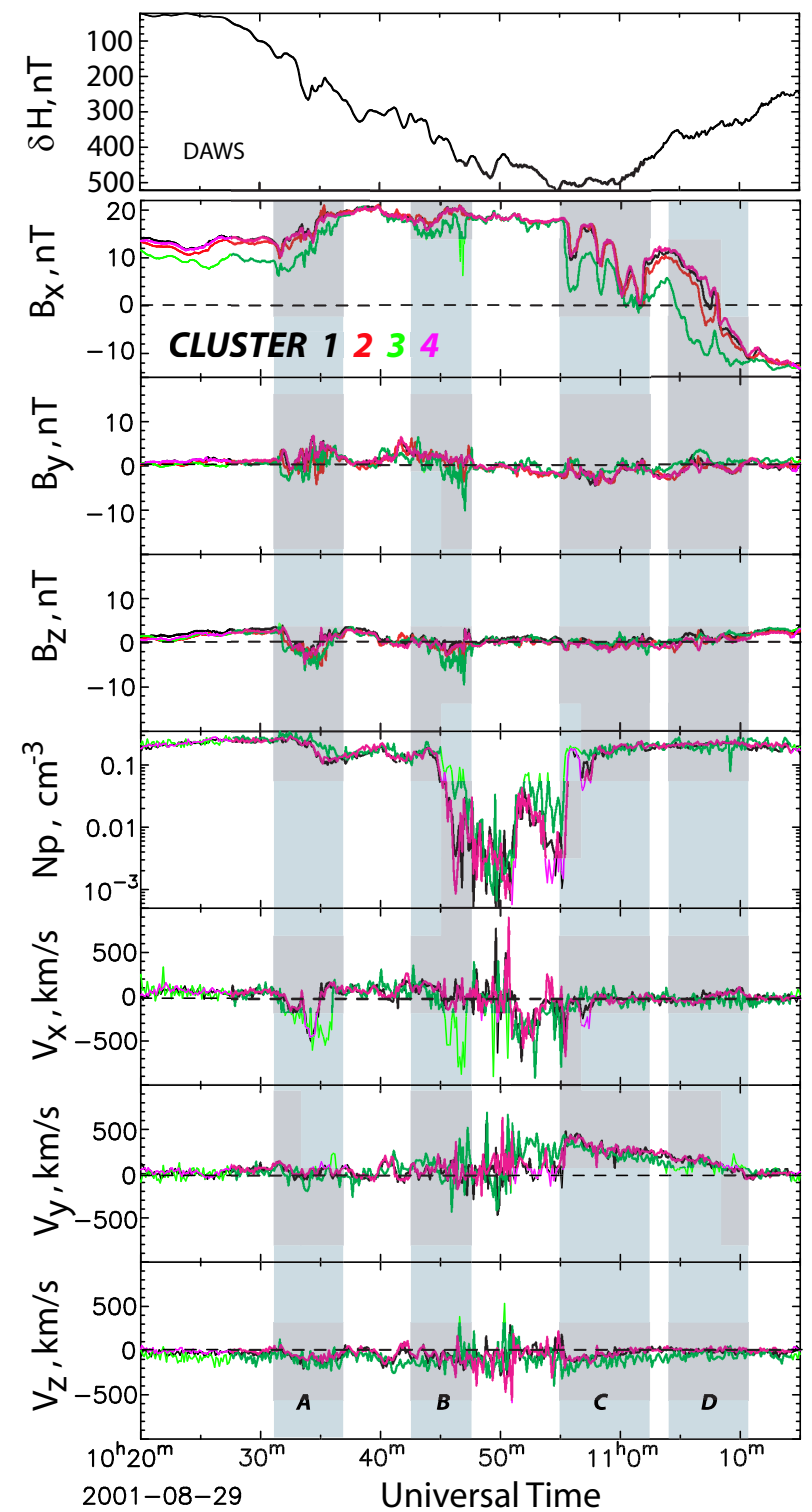

Fig. 3. CANOPUS (Dawson) magnetogram and Cluster/FGM and CIS-CODIF measurements in magnetotail during 10:20-11:15 UT, 29 August 2001.

(not shown), located near midnight. The energy of electrons, observed by LANL 1991-080, jumps from $\sim 500 \mathrm{eV}$ to $\sim 10 \mathrm{keV}$ at $\sim 10: 35 \mathrm{UT}$ and remains at this level until $\sim 11: 30$ UT.

Figure 3 shows the magnetic field, proton density and bulk velocity from Cluster/FGM (Balogh et al., 2001) and CIS-CODIF (Rème et al., 2001) together with the Dawson magnetometer record for the interval 10:20-11:15 UT. Calculated $X$ and $Y$ components of the magnetic field vertical gradient, which are differences between $X$ and $Y$ magnetic field components, measured by three northern s/c (\#1, 2, 4) and the s/c 3, $\left(D B_{x}\right.$ and $\left.D B_{y}\right)$, divided by the distance between three northern spacecraft and s/c $3(D Z=1700 \mathrm{~km})$; magnetic, plasma $\left(\mathrm{H}^{+}\right.$only), and total pressures and proton $\beta$ for this interval are shown in Fig. 4.

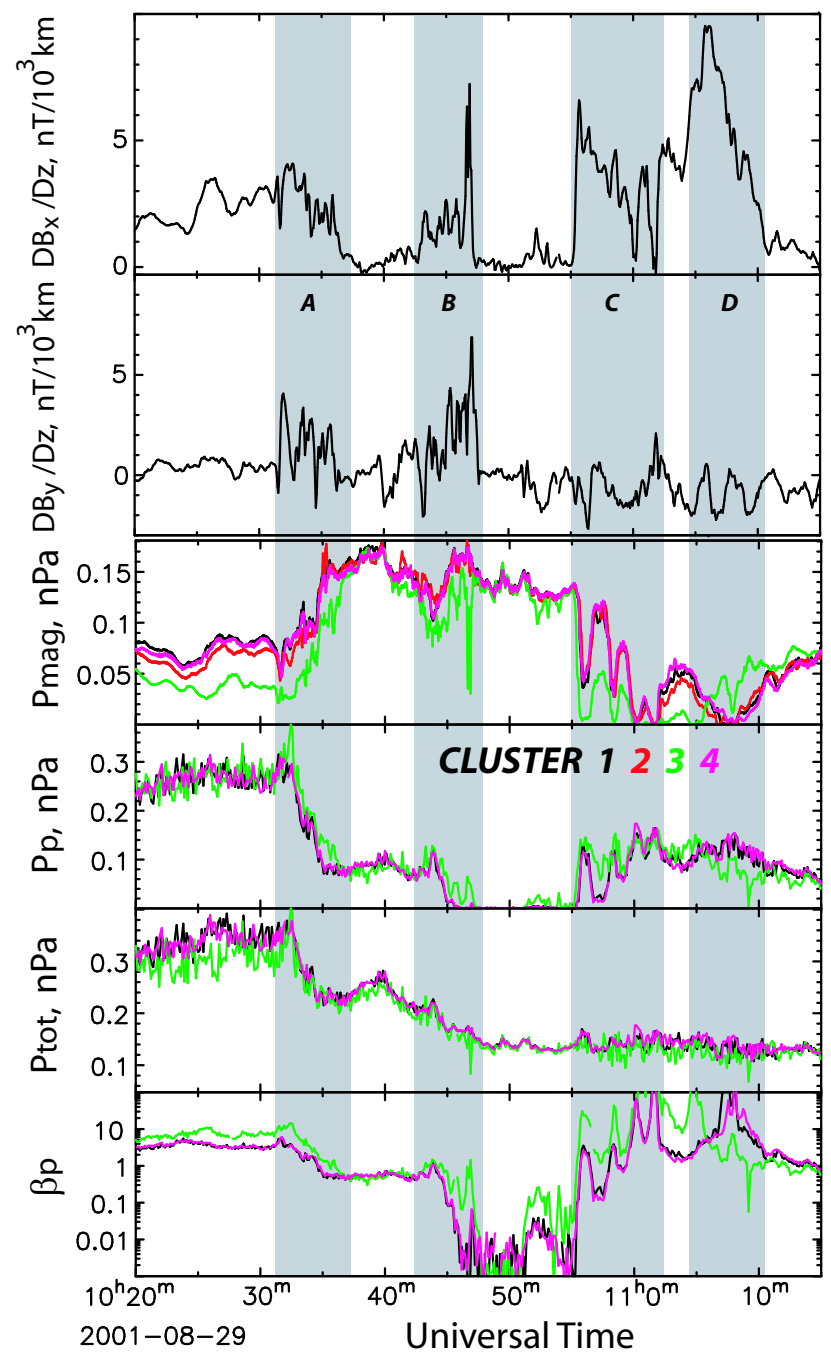

Fig. 4. Time variations of $X$ - and $Y$-components of the magnetic field vertical gradient, magnetic, plasma and total pressures, and plasma- $\beta$ parameter during 10:20-11:15 UT 29 August 2001.

At 10:28 UT (the electrojet onset time) Cluster was located in the central plasma sheet $\left(B_{x} \sim 10-13 \mathrm{nT}, N_{p} \sim 0.3 \mathrm{~cm}^{3}\right.$, $\left.\beta_{p} \sim 3-5\right)$. The $D B_{x} / D Z$, which is a proxy for cross-tail current density, varies from 2 to $4 \mathrm{nT} / 10^{3} \mathrm{~km}$. Starting at $\sim$ 10:31 UT (interval $A$ ) Cluster detects signatures of plasmoid and post-plasmoid flow: negative variation of $B_{z}$ followed by an $\sim 7 \mathrm{~min}$ long interval of negative $B_{z}$ with minimum value of $-5 \mathrm{nT}$ and negative $V_{x}$ about $-500 \mathrm{~km} / \mathrm{s}$. During interval $A$ the total and plasma pressures drop to 0.1 and $0.2 \mathrm{nPa}$, respectively, magnetic pressure increases up to $0.15 \mathrm{nPa}$, and $\beta_{p} \sim 0.9$.

At $\sim 10: 46$ UT (interval $B$ ) a negative variation of $X$ and $Y$ magnetic field components, associated with tailward proton flow with velocity of $-800 \mathrm{~km} / \mathrm{s}$, were detected by the most southern s/c 3. At the same time the other three spacecraft detect no plasma flow and a magnetic field of $20 \mathrm{nT}$. The $D B_{x} / D Z$ is $\sim 6-7 \mathrm{nT} / 10^{3} \mathrm{~km}$, which corresponds to a current density of about $4 \mathrm{nA} / \mathrm{m}^{2}$. The value of $D B_{y} / D Z$ is also about $6 \mathrm{nT} / 10^{3} \mathrm{~km}$, thus the current has a field-aligned 


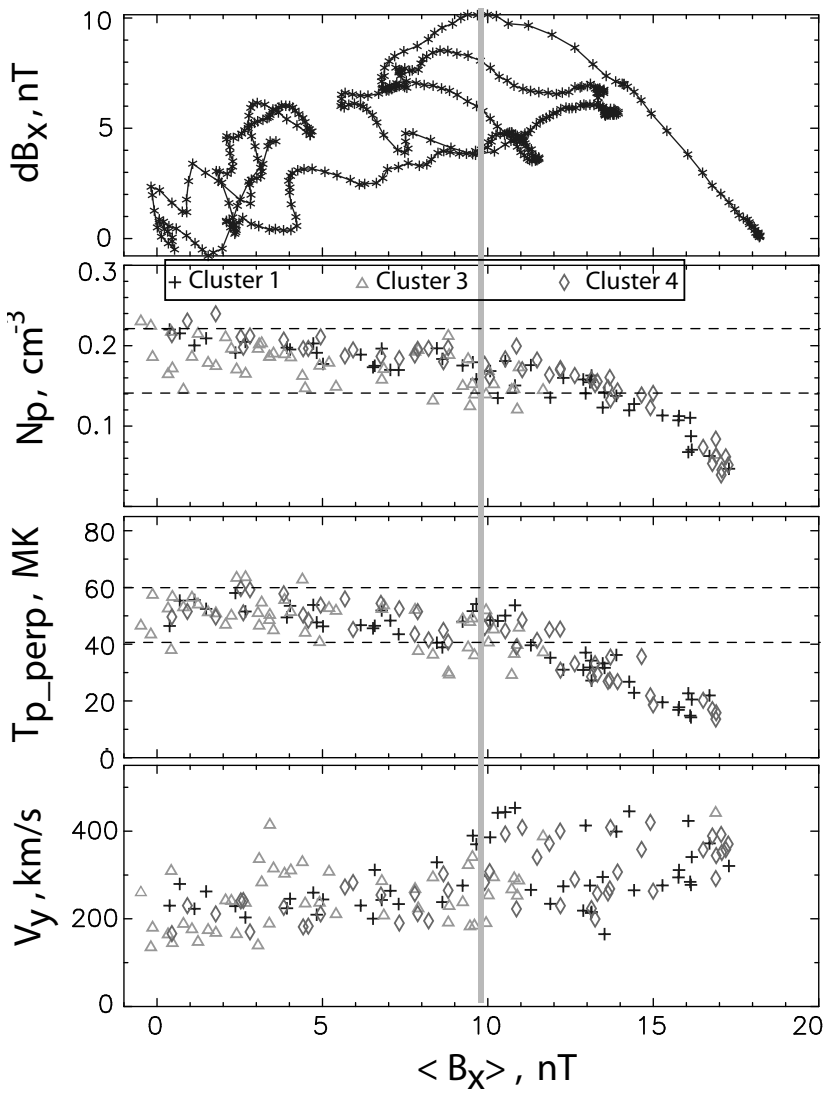

Fig. 5. Distributions of magnetic field difference $\left(D B_{x}\right)$, plasma density, perpendicular temperature and $Y$-component of bulk velocity versus $\left\langle B_{x}\right\rangle$ during interval $C$ (see explanations in the text).

component. Just after the detection of the strong magnetic field gradient the $\beta_{p}$ drops from about 1 to values of $10^{-3}$, the proton density decreases abruptly from $0.1 \mathrm{~cm}^{-3}$ to $\sim 0.005 \mathrm{~cm}^{-3}$, which indicates that Cluster passed from the plasma sheet into the northern magnetotail lobe, crossing the intensive current at the PSBL-lobe boundary.

At about 10:55 UT (interval $C$ ), at the peak of $\delta H$ on Dawson record, a drop in the magnetic field, a jump in the plasma density to $\sim 0.2$ and a jump in the $\beta_{p}$ from $\sim 0.005$ up to 10 were detected by all four spacecraft, which indicates an entrance to the plasma sheet. The $D B_{x} / D Z$ also jumps at 10:55:00-10:55:30 UT up to $7 \mathrm{nT} / 10^{3} \mathrm{~km}\left(\sim 4 \mathrm{nA} / \mathrm{m}^{2}\right)$. At the same time, the $D B_{y} / D Z$ shows no significant variation, thus the current is directed mostly in the dawn-to-dusk $\left(Y_{\mathrm{GSM}}\right)$ direction. The $Y$-component of the proton bulk velocity increases quickly up to $400 \mathrm{~km} / \mathrm{s}$. The total pressure is nearly conserved across this discontinuity.

During interval $C$ Cluster detects a set of negative variations in $B_{x}$ and an enhanced duskward proton bulk flow with the velocity of 500-300 $\mathrm{kms}^{-1}$. Time variations of $D B_{x} / D Z$ (Fig. 4) show that the current density is larger near the current sheet-lobe boundary than in the centre $\left(B_{x}=0\right)$. As was shown by Runov et al. (2003) the current sheet has a bifurcated structure with off-center maximum of the vertical gradient of the magnetic field during this interval. It was also found that the set of $B_{x}$ variations during interval $C$ is caused by waves propagating in the $+Y$ direction polarized in $Y-Z$ plane. Normal vector rotations were found in a range of 30 $35^{\circ}$.

During interval $D$ Cluster slowly crossed the neutral sheet. The spatial structure of the magnetic field gradient during this interval was studied by Runov et al. (2003). It was found that the current density has a maximum value at $B_{x} \sim 0$, which is expected from standard models.

It is worth noting that from $\sim 10: 30: 00$ to $10: 55: 30 \mathrm{UT}$ the tailward bulk proton flow with the velocity of $-500 \mathrm{~km} / \mathrm{s}$ was detected each time a spacecraft enters the plasma sheet $\left(\beta_{p} \sim 1\right)$. At the beginning of interval $C$ the character of the plasma flow changes abruptly. The tailward flow with $V_{x} \sim-600 \mathrm{~km} / \mathrm{s}$, detected at 10:55 UT when Cluster enters the current sheet, terminates at 10:55:30 UT, and a dawn-todusk intensive flow with a small $Z$-component started. This proton flow gradually decreases over intervals $C$ and $D$ and passes away at $\sim 11: 10 \mathrm{UT}$.

\section{Ion properties within the bifurcated current sheet}

It was shown by the analysis of the FGM data in our previous paper (Runov et al., 2003) that the vertical gradient of the magnetic field (which is the main part of the cross-tail current) has a maximum value at $\left\langle B_{x}\right\rangle \sim 0.5 B_{L}$, where $\left\langle B_{x}\right\rangle$ is the mean magnetic field of the northern group of $\mathrm{s} / \mathrm{c}$ (\#1, $2,4)$ and the most southern one (s/c, 3). This structure with off-center current density maximum was interpreted as the bifurcated or double-peak current sheet.

Figure 5 shows the difference between $\left\langle B_{x}\right\rangle_{124}$ (mean value from the northern group of spacecraft) and $B_{x 3}$, proton number density, proton temperatures parallel and perpendicular to the magnetic field, and $Y$-component of the proton bulk velocity from Cluster/CIS-CODIF versus $\left\langle B_{x}\right\rangle=0.5\left(\left\langle B_{x}\right\rangle_{124}+B_{x 3}\right)$. We cut the profiles of density, temperature and velocity at $N=0.04 \mathrm{~cm}^{-3}$. The hodogram $D B_{x}$ versus $\left\langle B_{x}\right\rangle$ shows that during interval $C(\sim 7 \mathrm{~min})$ the current has an off-center maximum near $\left\langle B_{x}\right\rangle \sim 10 \mathrm{nT}$, and a deep minimum $\left(D B_{x} \sim 0\right)$ near $\left\langle B_{x}\right\rangle=0$.

The vertical profiles of the proton density from s/c 1, 3 and 4 show a plateau with $N_{p}$ varying between $0.14-0.22 \mathrm{~cm}^{-3}$ within the central part of the sheet $\left(0 \leq B_{x} \leq 10 \mathrm{nT}\right)$ and sharply decreasing at $B_{x} \geq 10 \mathrm{nT}$. Similar behaviour of the plasma density was observed by Sergeev et al. (2003) within the bifurcated current sheet (26 September 2001 event). In both cases the average plasma density in the central part of the current sheet is $\sim 0.2 \mathrm{~cm}^{-3}$, which is slightly lower than the average density of $0.3-0.4 \mathrm{~cm}^{-3}$ found by Baumjohann et al. (1989) from a statistical analysis of AMPTE/IRM spacecraft data within geocentric distances $10<\mathrm{R}<19 R_{E}$.

The average temperature within $0 \leq B_{x} \leq 10 \mathrm{nT}$ is $\sim 50 \mathrm{MK}$ $(\sim 5 \mathrm{keV})$, which is a typical average value in the central plasma sheet (Baumjohann et al., 1989). The perpendicular temperature has small spread and increases roughly linearly from $20 \mathrm{MK}(\sim 2 \mathrm{keV})$ at $B_{x}=15 \mathrm{nT}$ up to $50 \mathrm{MK}$ at 
$B_{x}=10 \mathrm{nT}$, has a nearly flat profile and varies within 40 $60 \mathrm{MK}$ at $0 \leq B_{x} \leq 10 \mathrm{nT}$. The parallel temperature has a large spread and is not shown.

The profile of the $Y$-component of the proton bulk velocity also shows a nearly flat (with a tendency to decrease with $B_{x} \rightarrow 0$ ) profile in the central part of the sheet. An average value $V_{y}$ at $0 \leq B_{x} \leq 10 \mathrm{nT}$ is of $200 \mathrm{~km} / \mathrm{s}$. Out of the central part, at $10<B_{x}<18 \mathrm{nT}$, the $V_{y}$ is spread within $200-500 \mathrm{~km} / \mathrm{s}$. Values and the spatial behaviour of $V_{y}$ in this case differ significantly from the average ion velocity in the central plasma sheet found by Baumjohann et al. (1989). According to their analysis, the ion velocity at $X<-14 R_{E}$ is roughly constant about $60-70 \mathrm{~km} / \mathrm{s}$.

\section{Discussion}

Two examples of the magnetotail current sheet bifurcation, found from Cluster observations during the 2001 tail season, show similar properties: off-center peaks of the magnetic field gradient, an extended layer of weak uniform magnetic field, and a plateau in the plasma density profile between the peaks of the gradient. Because the $X$-component of the proton bulk velocity is negligibly small in both cases, we can not directly attribute the bifurcation to magnetic reconnection, as it was done by Sergeev et al. (1993) and Hoshino et al. (1996).

As it was shown by Arzner and Scholer (2001) and by Asano (2001) current sheet bifurcation may be a feature of a post-plasmoid plasma sheet (PPPS). The time history of the event (Fig. 3) shows the signatures of plasmoid and postplasmoid tailward plasma flow during intervals $A, B$ and at the beginning of the interval $C$. Asano (2001) has shown that in PPPS ion flow, mostly tailward, also has a duskward component. In our case the proton flow is mostly duskward. Arzner and Scholer (2001) and Asano (2001) also showed that electrons are the main carriers of the current in PPPS, and an ion input to the total current is smaller. This difference between electron and ion motions produces the 3-D Hall current system. Observations show, however, only the crosstail component of the current density (see Fig. 4).

Recently, Sitnov et al. (2003) have shown that the generalization of the Harris model, assuming ion temperature anisotropy outside the current sheet, gives rise to an equilibrium configuration with a bifurcated current. They found that the formation of the bifurcated current sheet with a flat profile of plasma density becomes possible for $T_{\perp}>T_{\|}$(pancake ion distribution) with the anisotropy ratio $T_{\perp} / T_{\|} \sim 1.1-1.2$. Calculation results display the profiles of current and plasma densities similar to observations. The cross-tail ion velocity profile resulting from the calculation has a local maximum near $B_{x} \sim 0.7 B_{L}$ and has a tendency to decrease with $B_{x} \rightarrow 0$ (M. Sitnov, private communication, 2003), which is close to the observations. The anisotropy of $10-20 \%$ could not be, however, resolved from the temperature measurements to prove an adequacy of this model to the observation.
Another possible mechanism of a bifurcated current sheet formation is magnetic turbulence, concentrated in a quasineutral sheet (see, e.g. Greco et al., 2002, and references therein). A series of numerical experiments shows that a double humped profile of the current density appears if the magnetic fluctuations $\delta B>0.2 B_{0}$, where $B_{0}$ is the magnetic field outside the current sheet, and the normal component of the field $\left(b_{n}\right)$ is less than 0.07 . Ion density and ion temperature also display plateau-like profiles within the sheet. The $Y$-component of the ion bulk velocity, resulting from the simulations, shows a two-hump profile with a local minimum in the center of the sheet $\left(B_{x}=0\right)$. It was found that with increasing $b_{n}$, a larger $\delta B$ is required to obtain the current bifurcation. With growth of $b_{n}$ the "valley" between two peaks of current becomes less deep.

Properties of the magnetic turbulence during 10:5511:35 UT on 29 August were analyzed by Vörös et al. (2003). They calculated the Local Intermittence Measure (LIM) as an indicator of the magnetic field turbulent properties. The enhancement of a large scale (characteristic time scale $\tau=40 \mathrm{~s}$ ) was found during interval $C$. At the same time, the level of the small scale LIM ( $\tau=0.4$ ) remains on a base level, which means that the magnetic field fluctuations in this scale are negligible. The difference between large-scale and small-scale LIM indicates an absence of a turbulent cascade within this interval. We analyzed also the variability of magnetic field within the current sheet and have found that the standard deviation $\left(\sigma B_{d}\right)$ calculated for each $1 \mathrm{nT}$ bin, where $B_{d}$ is the detrended magnetic field, obtained by subtraction of linear trend from the original magnetic field time series for each $8 \mathrm{~s}$ interval, has a tendency to increase towards the current sheet center $\left(B_{x}=0\right)$, but the ratio $\sigma B_{d} / B_{0}$, where $B_{0}$ is the mean magnetic field, calculated for the whole interval $C$ for all four spacecraft, is about 0.1 , which is less than the model requirement for the observed normal component $b_{n} \sim 0.02$.

The proton flow with large $V_{y}$, detected within intervals $C$ and $D$ may cause the Kelvin-Helmholtz (KH) instability, which results in the set of the magnetic field variations during the interval $C$ (Nakagawa and Nishida, 1989; Yoon et al., 1996). In this case the total pressure fluctuations $\delta P_{\text {tot }}$ are expected to be of $0.5 P_{t o t}$ (Yoon et al., 1996), which is not confirmed by the observation (Fig. 4). Karimbadi et al. (2003) showed by full particle and hybrid simulations that the velocity shear, resulting from the presence of secondary ion population, drives the ion-ion kink (IIK) mode instability, which produces $B_{x}$ variations similar to the observed, and a displacement of the current peak from the sheet center to lobes. The calculated wavelength $\left(2.8 R_{E}\right)$ and period $(90 \mathrm{~s})$ are close to the values which were found by Runov et al. (2003) and Sergeev et al. (2003).

\section{Conclusions}

We analyzed the evolution of plasma/current sheet structure for the isolated substorm on 10:20-11:15 UT, 29 August 
2001, using magnetic field and plasma data from Cluster spacecraft, located mostly in the central plasma sheet. It was found that during $\sim 7$ min around an $A E$ peak the current sheet had a bifurcated structure with the off-center current density maxima and an extended layer of a weak uniform magnetic field in the vicinity of $B_{x}=0$. The analysis of plasma data during this interval showed that plasma density, as well as plasma temperature, had a plateau-like profile in the central layer of the bifurcated current sheet and abruptly decrease at the plasma sheet/lobe boundary, which is associated with the layer of current density enhancement.

The extraordinary result is that current sheet bifurcation was associated with enhanced proton bulk velocity directed in $+Y_{\mathrm{GSM}}$ with a maximal value of $400 \mathrm{~km} / \mathrm{s}$. Other components of the proton velocity are negligibly small. An origin of this proton flow, which may be a cause of KH and IIK instabilities, remains unclear. Because no tailward or earthward strong flow was detected, we cannot directly attribute the current sheet bifurcation to the magnetic reconnection process. We discussed alternative mechanisms of a bifurcated sheet formation, based on ion anisotropy (Sitnov et al., 2003) and on magnetic turbulence, concentrated in the central part of the sheet (Greco et al. , 2002). Both models give results similar to observations, but the conditions required by the models are not completely satisfied in the considered case.

Acknowledgements. We would like to acknowledge the Cluster Science Data System (CSDS). We thank the Canadian Space Agency and J. Samson for the data from CANOPUS network. The data from ACE and GOES are available via CDAWEB. We used geosynchronous energetic particles monitor on the Leadbelly web-site. We thanks L. Zelenyi, M. Sitnov, H. Malova, G. Zimbardo and M. Prakash for fruitful discussions. This work was partly supported by RFBR N 03-02-17533 and N 03-05-20012, by WTZA AustriaRussia N I.1/04, and INTAS 03-51-3738 grants.

Topical Editor T. Pulkkinen thanks A. Taktakishvili for his help in evaluating this paper.

\section{References}

Arzner, K. and Scholer, M.: Kinetic structure of the post plasmoid plasma sheet during magnetotail reconnection, J. Geophys. Res., 106, 3827-3844, 2001.

Asano, Y.: Configuration of the thin current sheet in substorms, $\mathrm{PhD}$ Thesis, University of Tokio, 2001.

Asano, Y., Mukai, T., Hosino, M., Saito, Y., Hayakawa, H., and Nagai, T.: Evolution of the thin current sheet in a substorm observed by Geotail, J. Geophys. Res., 108, 1189, doi:10.1029/2002JA009785, 2003.

Balogh, A., Carr, C. M., Acuña, M. H. et al.: The Cluster magnetic field investigation: overview of in-flight performance and initial results, Ann. Geophys., 19, 1207-1217, 2001.

Baumjohann, W., Paschmann, G., and Catell, C. A.: Average plasma properties in the central plasma sheet, J. Geophys. Res., 94, 6597-6606, 1989.
Delcourt, D. C. and Belmond, G.: Ion dynamics at the earthward termination of the magnetotail current sheet, J. Geophys. Res., 103, 4605-4613, 1998.

Greco, A., Taktakishvili, A. L., Zimbardo, G., Vetri, P., and Zelenyi, L. M.: Ion dynamics in the near-Earth magnetotail: Magnetic turbulence versus normal component of the average magnetic field, J. Geophys. Res., 107, 1267, doi:10.1029/2002JA009270, 2002.

Harris, E. G.: On a plasma sheet separating regions of oppositly directed magnetic field, Nuovo Cimento, 23, 115-121, 1962.

Hoshino, M., Nishida, A., Mukai, T., Saito, Y., Yamamoto, T. and Kokubun, S.: Structure of plasma sheet in magnetotail: Doublepeaked electric current sheet, J. Geophys. Res., 101, 2477524786, 1996.

Karimbadi, H., Pritchett, P. L., Daughton, W. and KraussVarban, D.: Ion-ion kink instability in the magnetotail: 2 Three-dimensional full particle and hybrid simulations and comparison with observations J. Geophys. Res., 108, 1401, doi:10.1029/2003JA010109, 2003.

Nakagawa, T., and Nishida, A.: Southward magnetic field in the neutral sheet produced by wavy motions propagating in the dawn-dusk direction, Geophys. Res. Lett., 16(11), 1265-1268, 1989.

Nakamura, R., Baumjohann, W., Runov, A., Volwerk, M., Zhang, T. L., Klecker, B., Bogdanova, Y., Roux, A., Balogh, A., Rème, H., Sauvaud, J. A., and Frey, H. U.: Fast flows during current sheet thinning, Geophys. Res. Lett., 29(29), 2140, doi:10.1029/2002GL016200, 2002.

Rème, H., Aoustin, C., Bosqued, J. M. et al.: First multi-spacecraft ion measurements in and near the Earth's magnetosphere with the identical Cluster ion spectrometry (CIS) experiment, Ann. Geophys., 19, 1303-1354, 2001.

Runov, A., Nakamura, R., Baumjohann, W., Zhang, T. L., Volwerk, M., Eichelberger, H.-U., and Balogh, A.: Geophys. Res. Lett., 30 (2) 1036, doi:10.1029/2002GL016136, 2003.

Sergeev, V. A., Mitchell, D. G., Russell, C. T., and Williams, D. J.: Structure of the tail plasma/current sheet at $\sim 11 R_{E}$ ant its changes in the course of a substorm, J. Geophys. Res., 98, 17345-17365, 1993.

Sergeev, V., Runov, A., Baumjohann, W., Nakamura, R., Zhang, T. L., Volwerk, M., Balogh, A., Rème, H., Sauvaud, J. A., André, M., and Klecker, B.: Current sheet flapping motion and structure observed by Cluster, Geophys. Res. Lett., 30(6), 1327, doi:10.1029/2002GL016500, 2003.

Sitnov, M. I., Guzdar, P. N., Swisdak, M.: A model of the bifurcated current sheet, Geophys. Res. Lett., 30(13), 1712, doi:10.1029/2003GL017218, 2003.

Sitnov, M., Zelenyi, L. M., Malova, H. V., and Sharma, A. S.: Thin current sheet embedded within a thicker plasma sheet: Selfconsistent kinetic theory, J. Geophys. Res., 105, 13 029-13 043, 2000.

Vörös, Z., Baumjohann, W., Nakamura, R., Runov, A., Zhang, T. L., Volwerk, M., Eichelberger, H.-U., Balogh, A., Horbury, T. S., Glaßmeier, K.-H., Klecker, B., and Rème, H.: Multi-scale magnetic field intermittence in the plasma sheet, Ann. Geophys, 21, 1955-1964, 2003.

Yoon, P. H., Drake, J. F., and Lui, A. T.: Theory and simulation of Kelvin-Helmholtz instability in the geomagnetic tail, J. Geophys. Res., 101, 27 327-27 339, 1996. 